\title{
A Method of Insulator's NSDD Measurement and Its Device Based on Laser Transmission Principle*
}

\author{
Yang Liu', Song Gao', Jie Chen', Yanlong Lu ${ }^{2}$ \\ ${ }^{1}$ State Grid Jiangsu Electric Power Research Institute, Nanjing, China \\ ${ }^{2}$ Wuhan Canneng Electric Power Science and Technology Company Limited, Wuhan, China \\ Email: 15105168820@163.com
}

Received January 2014

\begin{abstract}
Accuracy measurement of the Non-soluble Deposit Density (NSDD) on the insulator surface is very important for the transmission line anti-pollution flashover works. A method to measure the NSDD on double sheds porcelain insulator surface based on laser transmission principle is proposed in this paper. Laser unit and luminous intensity sensor are installed between the up and down surface of the double sheds porcelain insulators, two glass tablets are put between the double sheds. The contamination on the glass tablets will influence the luminous intensity that reaches the intensity sensor. The luminous signal is changed to electrical signal, and the insulator's NSDD could be obtained based on the difference of luminous intensity. The device can be used in online monitoring of the insulator's NSDD condition on the insulator surface.
\end{abstract}

\section{Keywords}

Laser Transmission; NSDD; Luminous Intensity; Real-Time Measurement; Device

\section{Introduction}

Insulator pollution flashover is one of the important problems which influence the safety and stability of power system. Insulators of overhead transmission line operated under natural condition in long term and the surface is polluted by conductive and non-conductive materials in the air. In wet weather conditions such as fog, dew, rain, thaw and drizzle, the insulation performance of insulators significantly reduced which may lead to pollution flashover and cause tripping under rated operation voltage. Due to the similarity of pollution condition and the similarity of meteorologic condition in specific region, the success rate of reclosing after pollution flashover is low and the failure of reclosing may cause line outage. Frequent occurrence of pollution flashover may lead to large area blackout which may seriously endanger the safety and stable operation of the power system [1] [2]. The number of accidents caused by pollution flashover is the second major factor of total accidents of power system which is second only to lightning failures. But the economic loss caused by pollution flashover is about 10 times of that caused by lightning failures [3].

*Project supported by Science and Technology Project of State Grid Jiangsu Power Company (J2013010).

How to cite this paper: Liu, Y., Gao, S., Chen, J. and Lu, Y.L. (2014) A Method of Insulator's NSDD Measurement and Its Device Based on Laser Transmission Principle. Journal of Power and Energy Engineering, 2, 518-524.

http://dx.doi.org/10.4236/ipee.2014.24070 
Polluting, wetting, formation of dry bands and partial arc, development from arc to completely flashover are the four parts in the process of pollution flashover. The wetting process is an important factor because it determines the conductivity of contamination layer on the insulator surface. The NSDD directly determine the saturated moisture water content and the pollution withstand voltage of insulators is also determined.

The measuring methods of pollution degree are mainly focusing on leakage current $[4,5]$, equivalent salt deposit density (ESDD) [6,7], pollution layer conductivity [8,9] and surface flashover electric field strength [10]. But some of the measuring methods belong to indirect measurement and others need the disassembling of the insulators. Thus the process of these methods is complex and is hard to apply to online monitoring of NSDD. Some non-contact measuring methods are put forward, such as infrared temperature measurement [11]. But this method is based on back propagation neural network. The measurement results are significantly affected by the parameters of the model. Considering the characteristic of all methods above, a measurement method of NSDD on porcelain insulator surface based on the laser transmission principle and relevant device are proposed in this paper. The structure of the device is simple and the measurement result is easy to obtain. This method can be applied to online monitoring of NSDD.

\section{Measuring Principle and Devices}

Laser is unidirectional and convergent. When the laser beam passes through a glass tablet, part of the laser will reflect and the other part that passes through the glass tablet can keep good directivity. This feature of laser ensures that the luminous intensity sensor can receive enough luminous signal. The principle diagram and schematic diagram of measurement device is shown in Figures 1 and 2, respectively. The laser unit and the luminous intensity sensor are respectively installed on down surface of the upper insulator shed and the up surface of the lower shed. Sampling glass tablet 1 and sampling glass tablet 2 can respectively collect the filth of the two sheds. The filth collected in the two sampling glass tablets can equivalently reflect the density of contamination on the insulator surface. As the sampling glass are gradually contaminated, the luminous intensity that reach the intensity sensor decreases. Comparing the luminous density signal and the fitted curve of luminous intensity-NSDD which is calibrated in the laboratory in advance, the NSDD status of the test insulator is obtained.

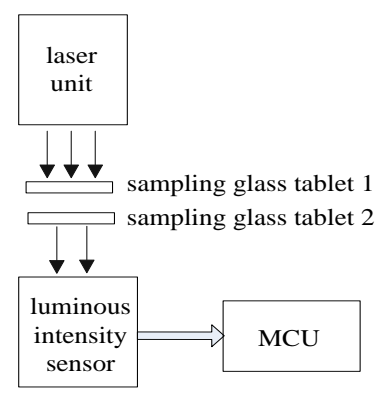

Figure 1. Schematic diagram of measurement device.

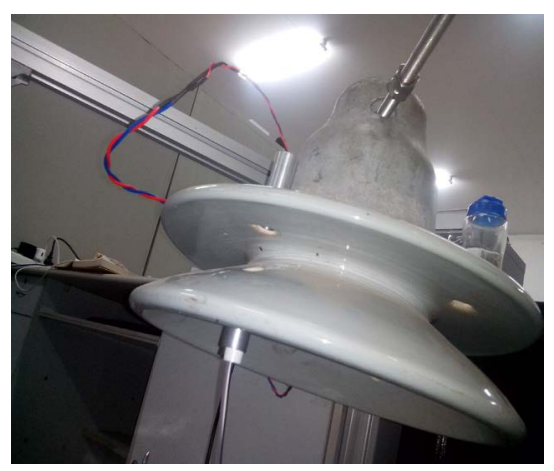

Figure 2. Factual picture of measurement device. 
According to GB/T4585-2004 (artificial pollution tests on high-voltage insulators to be used on a.c. systems standard) of China and Q/GDW152-2006 (pollution classification and external insulation selection for electric power system standard) of SGCC of China, NSDD between $0.25 \mathrm{mg} / \mathrm{cm}^{2}$ and $4.0 \mathrm{mg} / \mathrm{cm}^{2}$ can be divided into 10 grades. And calibration glass tablet is made in laboratory for testing the luminous intensity.

In order to eliminate the interference of background light in natural environment, the testing time of NSDD is recommended to choose at night.

\section{Measuring Device Module}

\subsection{Laser Units}

Wavelength used in the laser unit is $650 \mathrm{~nm}$. The power of laser module is $20 \mathrm{~mW}$. Factual picture of laser unit is shown in Figure 3.

The luminous intensity of laser module is generally large. In order to keep the luminous intensity within the measurement range of sensor, the peripheral circuit in the laser module of the device is redesigned. The improved circuit is shown in Figure 4.

The circuit can change the luminous density of the laser conveniently so that the initial value is within the collection range of the luminous intensity sensor. Meanwhile, in order to reduce the power consumption of the whole system circuit, MCU is used to control the switch of the laser tube. The laser tube only operates when the measuring process starts.

\subsection{Luminous Intensity Acquisition Circuit}

BH1750FVI is chosen to be the luminous intensity acquisition circuit [12]. BH1750FVI is an integrated circuit used in two wire serial bus interface. The circuit can adjust the brightness of LCD and keyboard backlight (Tabel 1).

According to the collected data of luminous intensity, with the high resolution of the circuit, wide range of luminous intensity change can be detected. And the value is between 1lx-65535lx. According to the handbook of the chip, the luminous intensity acquisition circuit is designed as Figure 5.

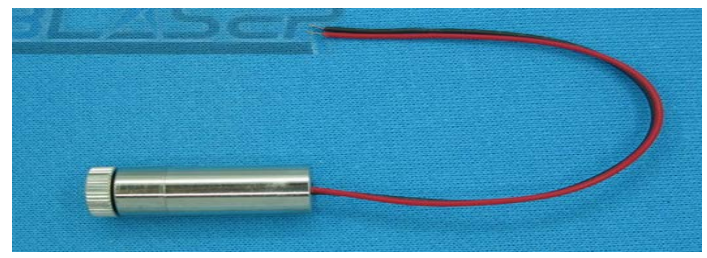

Figure 3. Factual picture of laser unit.

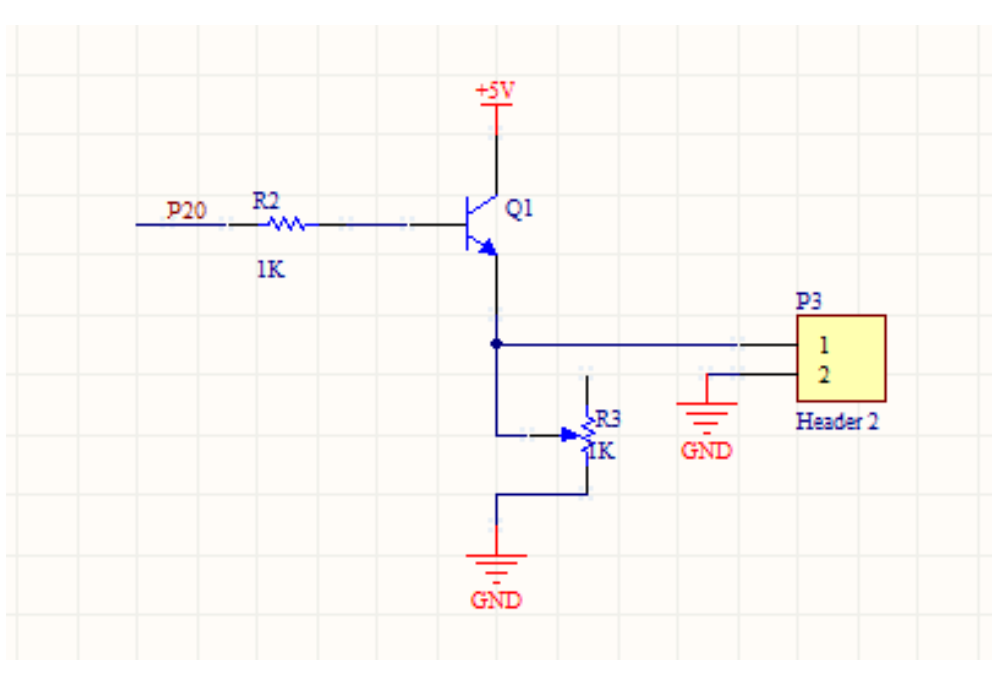

Figure 4. Circuit of laser unit. 


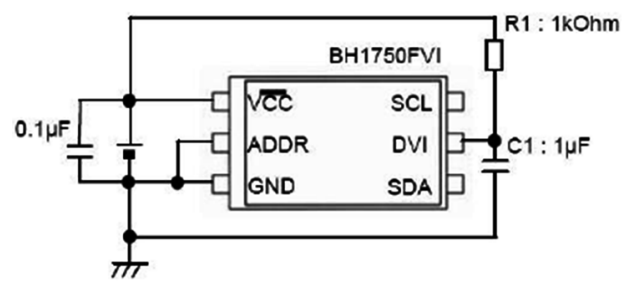

Figure 5. Luminous intensity acquisition circuit.

Table 1. Diameters of laser unit.

\begin{tabular}{cccc}
\hline Item & Value & Item & Value \\
\hline output wavelength & $655 \pm 3 \mathrm{~nm}$ & connection method & common cathode \\
output power & $20 \mathrm{~mW}$ & Starting time & $\leq 0.01$ \\
working voltage & $2.7-4.0 \mathrm{~V}$ & Service life & $>6000 \mathrm{~h}$ \\
working current & $<70 \mathrm{~mA}$ & boundary dimension & $\Phi 12 * 35 \mathrm{~mm}$ \\
Working temperature & $-10^{\circ} \mathrm{C}-45^{\circ} \mathrm{C}$ & texture & copper \\
storage temperature & $-40^{\circ} \mathrm{C}-80^{\circ} \mathrm{C}$ & color & silvery \\
focal spot & adjustable & Net weight & $12.5 \mathrm{~g}$ \\
spot diameter & adjustable $\leq \Phi \mathrm{mm}$ & Directing of rotation & $\mathrm{CW}$ laser \\
\hline
\end{tabular}

\section{Main Circuit of MCU}

\subsection{Design of the Circuit}

STC12 single chip is used as the MCU. STC series integrated MCU, A/D converter, E2PROM and other modules. Thus, the peripheral devices of corresponding chip can be left out. It reduces the difficulty of circuit design and software programming [13]. The main circuit of MCU is shown in Figure 6.

The circuit operates as the follow principle. MCU controls the switch of the laser tube to control which determine whether the system begins to collect data. When the laser tube is off, the system is in low power state. The system power consumption is reduced so that the life time of the system is extended and the efficiency and durability of the system are improved.

\subsection{Monitoring Platform}

In order to get measurement values more conveniently and characterize the relationship between the change of NSDD and the luminous intensity, a visual monitoring platform in the master computer of the device is designed with Visual Basic software specially. The interface of the platform is shown in Figure 7.

With the platform, the real-time changes of the luminous intensity can be monitored and recorded. Changing NSDD of the up surface and the down surface of the middle layer, the corresponding change of the luminous intensity is recorded in the platform. With these data, a relation curve is formed in the platform.

\section{Measuring and Testing}

\subsection{Measurement Methods and Steps}

After the construction of the detection and monitoring platform is completed, the glass sheets can be calibrated to verify the effectiveness of the device. There is no NSDD measuring samples for sale in the market, hence NSDD measuring samples need to be calibrated. Production and calibrating methods are as follows.

Intercept a group of glass sheets of the same area, thickness and material as a backup.

Take respectively a certain amount of diatomite according to $0.25 \mathrm{mg} / \mathrm{cm}^{2}-4.0 \mathrm{mg} / \mathrm{cm}^{2}$ NSDD and dissolve the diatomite in a certain volume of water.

Apply the solution evenly to the glass samples.

Repeat step (2) and step (3) to make calibration glass of 10 grades. The 10 grades are determined according to the NSDD ranging from $0.25 \mathrm{mg} / \mathrm{cm}^{2}$ to $4.0 \mathrm{mg} / \mathrm{cm}^{2}$. 


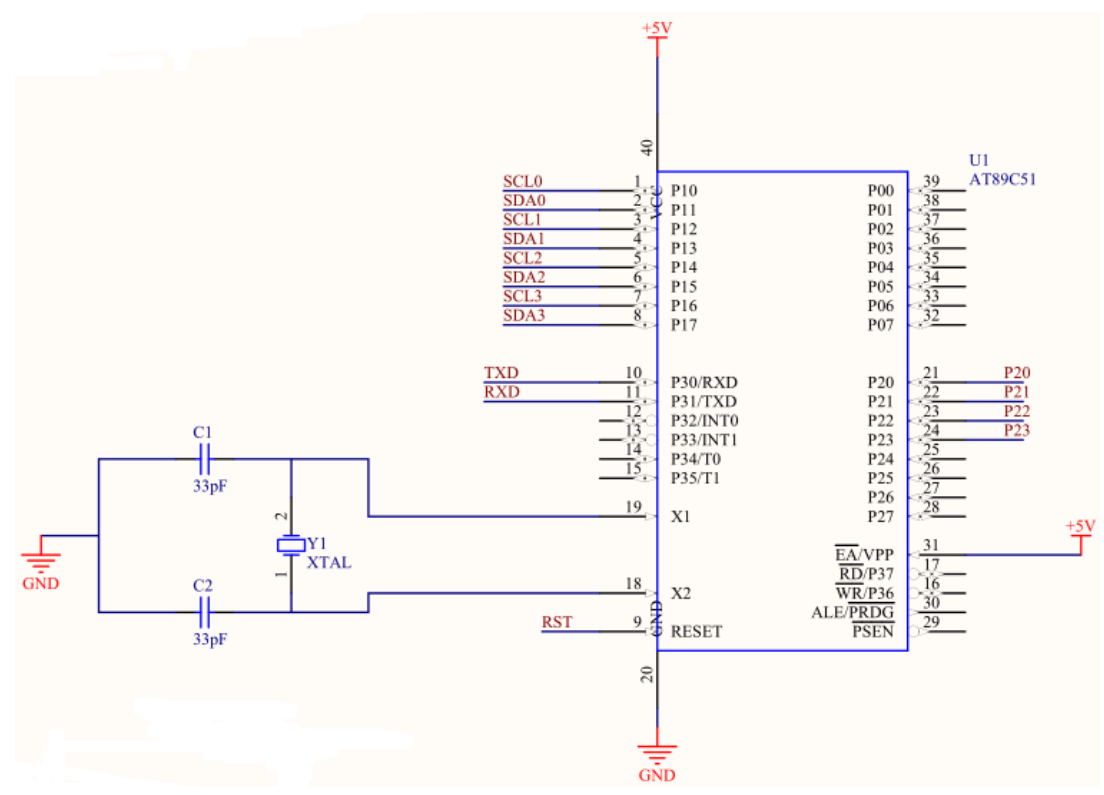

Figure 6. Main circuit of MCU.

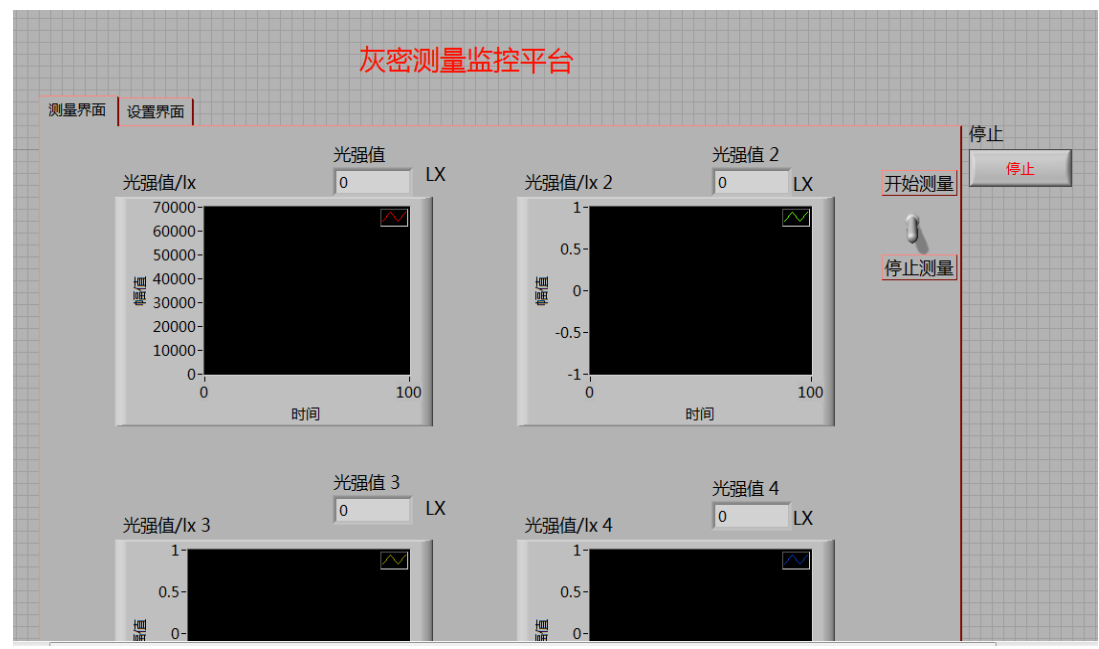

Figure 7. Monitoring platform interface.

When the calibration of glass is completed, the light transmittance intensity can be measured, and the measurement steps are as follows:

Start the laser tube and adjust the appropriate luminous intensity value, then note the initial value.

Put the NSDD sample of the first grade between the laser unit light and the receiver of luminous intensity sensor, record the data of luminous intensity when the number is stable measure all the NSDD samples as step (2).

Modeling and get and the corresponding changing relation between NSDD samples and the luminous intensity.

\subsection{Actual Measurement}

Set the initial value of the light intensity to about 46000LX. The interface of the monitoring platform is shown in Figure 8, and the luminous intensity reach maximum at this time point.

As shown in Figure 9, after putting into the NSDD samples in the device, the values of luminous intensity changes significantly on the monitor. 


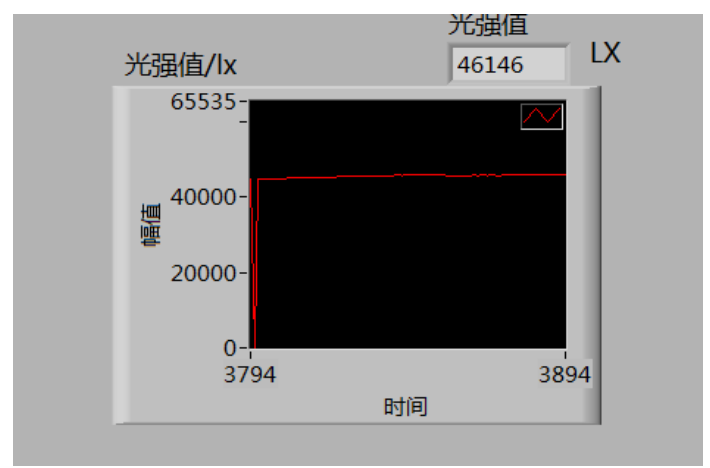

Figure 8. Initial luminous intensity value.

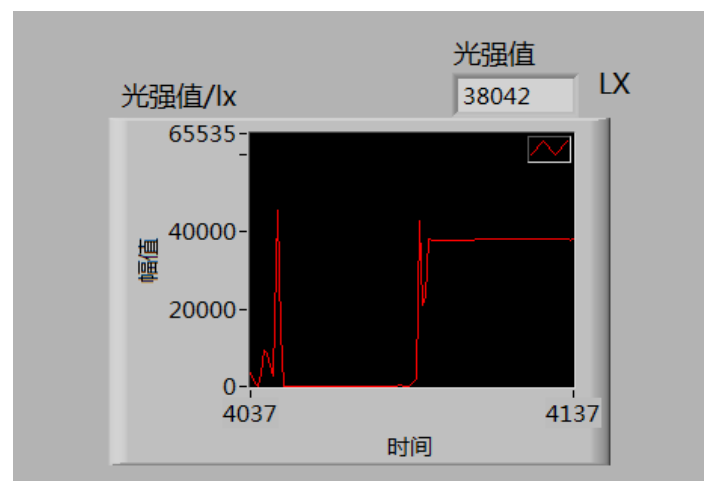

Figure 9. Intensity value of NSSD glass tablet.

\section{Conclusion}

In this paper, a method to measure the NSDD on double sheds porcelain insulator surface based on laser transmission principle is proposed. The design chart and relevant circuit is also demonstrated. Rationality and reliability of the method are verified according to the actual measurement data.

\section{References}

[1] Guan, Z.C., Liu, Y.Y. and Zhou, Y.X. (2006) External Insulation of Insulator and Electric Transmission and Transformation Equipment. Tsinghua University Press, Beijing.

[2] Gu, L.G. and Sun, C.X. (1990) Pollution Insulation of Electric Power System. Chongqing University Press, Chongqing.

[3] Zhang, Z.J., Jiang, X.L. and Sun, C.X. (2006) Present Situation and Prospect of Research on Flashover Characteristics of Polluted Insulators. Power System Technology, 30, 35-40.

[4] Wang, L.M. and Li, B. (2010) Development of a 19-Channel Leakage Current Online Monitoring System for Natural Pollution Deposit Test. High Voltage Engineering, 36, 559-564.

[5] Kim, J.H. and Song, W.C. (2001) Leakage Current Monitoring and Outdoor Degradation of Silicone Rubber. IEEE Transactions on Dielectric and Electrical Insulation, 8, 1108-1115. http://dx.doi.org/10.1109/94.971471

[6] Chen, Y.Q., Wu, G.Y. and Tian, Q. (2001) Investigation of Salt Density in Substation and Transmission Line by Optic Spectrum in Laboratory. High Voltage Engineering, 20, 481-487.

[7] Chisholm, W.A. and Buchan, P.G. (1994) Accurate Measurement of Low Insulator Contamination Levels. IEEE Transactions on Power Delivery, 9, 1552-1557. http://dx.doi.org/10.1109/61.311211

[8] Zhang, R.Y. and Guan, Z.C. (1990) Conductivity of Partial Surface: A New Method to Describe Contaminated Degree of Insulator. High Voltage Engineering, 16, 20-26.

[9] Zhao, Z.Y., Zou, J.Y. and Wu, G.Y. (2000) On-Site Measurement of Pollution Layer Conductivity for Suspension Insulators. High Voltage Engineering, 26, 37-38.

[10] Shu, L.C. Yang, J.L. and Jiang, X.L. (2009) Effect of Pre-Polluting Methods on DC Flashover Characteristics of Arti- 
ficially Iced Porcelain and Glass Insulators. High Voltage Engineering, 35, 1294-1300.

[11] Reddy, B.S. (2003) Study of Temperature Distribution along an Artificially Polluted Insulator String. Plasma Science \& Technology, 5, 1715-1720. http://dx.doi.org/10.1088/1009-0630/5/2/006

[12] Yun, Z.H. and Bai, T.R. (2012) Indoor Light Intensity Measuring Instrument Based on BH1750FVI. Microcontrollers \& Embedded Systems, 6, 27-29.

[13] Xu, D., Dai, X.J. and Li, X.Y. (2012) Design of Digital Carbon Monoxide Concentration Transmitter Based on STC12C5608AD MCU. Machinery \& Electronics, 9. 